\title{
POTT'S PARAPLEGIA IN THE NIGERIAN
}

\author{
B. S. Jones, Cape Town, South Africa \\ From the Igbobi Orthopaedic Hospital, Lagos, Nigeria
}

Although most victims of Pott's paraplegia recover completely with conservative treatment alone, the tragedy of that small but significant percentage that remain permanently paralysed still presents a challenge.

The operation of antero-lateral decompression has earned a permanent place in the management of these cases, and Seddon (1956) has recently defined the indications for its employment, a fitting sequel to the classical papers in which he (1935) and Butler (1935) did so much to clarify the pathogenesis and prognosis of the condition.

The purpose of this paper is to review the cases of Pott's paraplegia treated in this hospital during six and a half years, with particular reference to the place of operative intervention.

\section{CLINICAL MATERIAL AND GENERAL DATA}

There were admitted to the Igbobi Orthopaedic Hospital, Lagos, between January 1949 and June 1955, 276 patients with tuberculosis of the spine, of whom 115 at one time or another suffered paraplegia or paraparesis. This unusually high percentage was due to a shortage of beds, which necessitated the treatment of most patients with uncomplicated tuberculous spinal disease as out-patients. All the patients were African. Of the paraplegic patients sixty were male and fifty-five female.

Eight patients died while in hospital, three from pressure sores and toxaemia, three from tuberculous meningitis, one from cerebral malaria and one from an unknown cause. Eighty-six patients recovered completely; ten recovered with minor residual effects; eleven were permanently paralysed, seven partially and four totally.

Age-The highest quinquennial incidence was at five to nine, and the second highest ten to fourteen (Table I).

Vertebral bodies affected - The sixth, seventh, eighth and ninth were the thoracic vertebrae affected most often (Table II). The average number involved in patients with paraplegia was 3.5 , in cases without paraplegia 3.4 (Table III).

Grade of paraplegia according to onset-The distribution of cases according to grade of paraplegia is shown in Table IV, grade 1 representing a spastic paraplegia in which some voluntary control is retained, grade 2 a severe paraplegia in extension with complete loss of voluntary movement, grade 3 a paraplegia in flexion and grade 4 a flaccid paraplegia.

\section{GRADES OF PARAPLEGIA}

\section{EARLY ONSET PARAPLEGIA}

Grade 1-There were twenty-five cases of early onset paraplegia of grade 1, of which nineteen persisted unchanged for up to six months, three for six to twelve months, one for twelve to twenty-four months, two for over twenty-four months. Twenty were treated by purely conservative means-that is, on alternated posterior and anterior plaster shells with (in all but the earliest cases) streptomycin twice weekly, supplemented by para-amino-salicylic acid alone at first and by sulphaniazid also in later cases for a course of three to six months.

Costo-transversectomy was undertaken in four patients and in one a paravertebral abscess was aspirated. Twenty-three patients recovered completely. One patient with a three years' history and with a severe kyphos and an apparently healed spinal lesion remained permanently 
POTT'S PARAPLEGIA IN THE NIGERIAN

TABLE I

Age (Years)

$\frac{0-4}{22} \frac{5-9}{40} \frac{10-14}{32} \frac{15-19}{10} \frac{20-29}{5} \frac{30-39}{4} \frac{40 \text { and over }}{2}$

TABLE II

Distribution

\begin{tabular}{|l} 
Vertebrae involved \\
Frequency .
\end{tabular}$\frac{\mathrm{C}}{3} \frac{\mathrm{T} .1}{6} \frac{\mathrm{T} .2}{11} \frac{\mathrm{T} .3}{22} \frac{\mathrm{T} .4}{28} \frac{\mathrm{T} .5}{38} \frac{\mathrm{T} .6}{46} \frac{\mathrm{T} .7}{49} \frac{\mathrm{T} .8}{49} \frac{\mathrm{T} .9}{43} \frac{\mathrm{T} .10}{37} \frac{\mathrm{T} .11}{30} \frac{\mathrm{T} .12}{20} \frac{\mathrm{L} .1}{13} \frac{\text { L.2 }}{6} \frac{\text { L.3 }}{4}$

TABLE III

Average Number of Vertebrae Involved

\begin{tabular}{|c|c|}
\hline Paraplegic cases & Non-paraplegic cases \\
\hline 3.5 & 3.4 \\
\hline
\end{tabular}

TABLE IV

Type and Grade of Paraplegia

\begin{tabular}{|l|ccc|c|c|}
\hline & Grade 1 & Grade 2 & Grade 3 & Grade 4 & Total \\
\hline Early onset & 25 & 56 & 11 & 10 & 102 \\
\hline Late onset & 4 & 5 & 3 & 1 & 13 \\
\hline
\end{tabular}

TABLE V

Correlation between Paraplegia and State of Abscess

\begin{tabular}{|lccc|c|}
\hline & $\begin{array}{c}\text { Bone } \\
\text { lesion } \\
\text { active }\end{array}$ & $\begin{array}{c}\text { Lesion } \\
\text { apparently } \\
\text { inactive }\end{array}$ & $\begin{array}{c}\text { Paravertebral } \\
\text { abscess }\end{array}$ & $\begin{array}{c}\text { Recovery of paraplegia } \\
\text { coincident with } \\
\text { absorption of abscess }\end{array}$ \\
\hline Early onset & 100 & 2 & 88 & 44 \\
\hline Late onset & 8 & 5 & 7 & 5 \\
\hline
\end{tabular}

TABLE VI

DURATION OF FOLLOW-UP

Follow-up $\frac{5 \text { years and over }}{9} \frac{4 \text { years }}{10} \frac{3 \text { years }}{26} \frac{2 \text { years }}{26} \frac{1 \text { year }}{28} \frac{\text { Less than } 1 \text { year }}{16}$

VOL. 40 B, NO. 1, FEBRUARY 1958 
paraplegic. Another patient with disease of almost three years' duration with an apparently healed spinal lesion but a persistent paravertebral abscess shadow and anterior scalloping of the vertebral bodies was treated by costo-transversectomy. Eighty cubic centimetres of inspissated pus were found retained beneath the anterior longitudinal ligament, and almost full recovery followed its release.

Grade 2-There were fifty-six patients with early onset paraplegia of grade 2, of whom forty-six recovered completely and ten did not. Of these ten, four (three treated entirely conservatively and one by an unproductive costo-transversectomy) recovered with only trifling residual paresis. Three, one subjected to an unproductive costo-transversectomy after three months, one to an unproductive costo-transversectomy and laminectomy after a year and one to antero-lateral decompression after three years, had permanent partial paraplegia. One patient, in whom release of a paravertebral abscess after four months and later antero-lateral decompression at twenty-three months were ineffective, ended with a complete permanent paraplegia of grade 3. One patient, rapidly deteriorating at nine months, did not improve after antero-lateral decompression and died five weeks later. One patient treated conservatively for seventeen months deteriorated to grade 3 and died five weeks later. One patient treated conservatively for seventeen months deteriorated to grade 3 and died with sores and toxaemia.

In all, twenty-seven patients were treated conservatively throughout; sixteen costotransversectomies, thirteen antero-lateral decompressions, three laminectomies and one aspiration of a paravertebral abscess were performed.

The duration of paraplegia before progressive improvement began was of prognostic import, and improvement was often attended by radiological evidence of diminution of a paravertebral abscess. Thus, of the 102 patients with early onset paraplegia, eighty-eight had a paravertebral abscess, and in forty-four of these improvement in the paraplegia closely coincided with absorption of the abscess (Table V). Only progressive improvement is significant. Incomplete paraplegia may vary in grade 1 and grade 2 for as long as two or three years; in such cases the paravertebral abscess persists. Further, even after progressive improvement relapse sometimes occurs. Of the forty-six patients who recovered completely, twenty-three began to improve within three months, twelve between three and six months, ten between six and ten months, and in one exceptional case a patient who had a large paravertebral abscess successfully evacuated by costo-transversectomy five months from the onset did not begin to improve till ten months later, when recovery became complete fairly rapidly.

Of the four patients showing only slight residual paresis, one fluctuated between grade 1 and grade 2 paraplegia for twenty months, one began to improve at two months but later relapsed and the remaining two began to improve at six and ten months. Of the four cases with permanent partial or complete paraplegia improvement began at thirteen months in one, two years in another and not at all in the remaining two.

In brief, it seems that in a case of early onset paraplegia of grade 2 progressive improvement within six months of the onset almost assures complete recovery; onset of progressive improvement at between six and ten months gives the prospect of residua in approaching 20 per cent; if progressive improvement is delayed for more than a year the paraplegia is likely to be permanently partial or complete.

Grade 3-There were eleven patients with early onset of paraplegia of grade 3. Of four with paraplegia of less than one month's duration complete recovery occurred in two treated conservatively, complete slow recovery in one after costo-transversectomy, and there were very slight residua in one after antero-lateral decompression.

Of four patients with paraplegia of from one to three months' duration two recovered completely after conservative measures and antero-lateral decompression respectively, one had slight residual paresis after antero-lateral decompression, and the fourth, showing progressive improvement after antero-lateral decompression, died three months later from cerebral malaria.

One patient showed no response to costo-transversectomy at two months, but began to 
improve with antibiotics at six months and recovered completely. One patient with paraplegia of fifteen months' duration, that had been of grade 3 for at least five months, was unaffected by costo-transversectomy and antero-lateral decompression and remained permanently paralysed. The last patient, with paraplegia of two years' duration, that had deteriorated to grade 3 for an undetermined period, regressed to a severe grade 1 with absorption of a small paravertebral abscess on conservative treatment.

Five patients were treated purely conservatively; five antero-lateral decompressions and four costo-transversectomies were performed.

In short, complete recovery appears the rule in early onset paraplegia of grade 3 that has persisted for less than one month, and recovery is probable when paraplegia has persisted for between one and three months. After three months there is a strong probability of significant residual paresis or of permanent paraplegia.

Grade 4-There were ten patients with early onset paraplegia of grade 4. Of four patients with paraplegia of not more than two weeks' duration one died from tuberculous meningitis, two recovered completely after antero-lateral decompression and one with primary disease of the neural arches recovered completely after laminectomy. Of three patients with paraplegia of between two weeks' and two months' duration two recovered completely, one with conservative measures, the other after antero-lateral decompression and the third had residual paresis after costo-transversectomy. Of three patients with paraplegia of four, seven and eight months' duration, two died from tuberculous meningitis (one three months after anterolateral decompression) and in the third, despite antero-lateral decompression, paraplegia remained complete and permanent. Two patients were treated purely conservatively, and five antero-lateral decompressions, one costo-transversectomy and one laminectomy were performed.

\section{LATE ONSET PARAPLEGIA}

There were thirteen cases of late onset paraplegia. Grading was often not so clear-cut as in cases of early onset because paralysis was often incomplete and physical signs were often irregular. Thus in a grade 2 early onset case the supervention of sensory loss, trophic sores, flexor spasms or sphincter disturbance fairly certainly heralds early deterioration to grade 3 or 4 . In late onset paraplegia on the other hand it is by no means rare to find retention of a significant degree of voluntary control attended by sensory loss, sphincter disturbance or trophic sores.

Nevertheless, the term late onset paraplegia embraces all cases of paralysis occurring for the first time more than two years from the onset of spinal disease. In fact four distinct types of late onset paraplegia were distinguished in this series, and the type of paraplegia was of more prognostic significance than its grade. The types are as follows.

Type a-In a case of spinal disease of perhaps three or four years' duration, often without extreme destruction or severe deformity, which was pursuing a slowly progressive course towards quiescence, probably under inadequate treatment, there occurs a recrudescence of activity attended by paraplegia. Radiographs show clear evidence of activity in the spinal lesion and usually a paravertebral abscess.

Type b-This is the type in which low-grade, grumbling spinal disease has been present for five to twenty years, and in which treatment has either never been sought or has been inadequate. A gross gibbus makes an angle of less than 90 degrees. Radiographs may show slight evidence of activity, but the disease is never florid and there is no paravertebral abscess, while mottled calcification in the lesion or at the site of an old abscess may indicate past attempts at healing. Some cases are intermediate between types (a) and (b).

Type c-Sometimes paraplegia supervenes upon a gross, long-standing kyphosis, but clinical, radiological and even operative findings show no sign of activity of the disease and suggest that the spinal lesion is in fact completely healed, but usually without bony fusion.

VOL. $40 \mathrm{~B}$, NO. 1, FEBRUARY 1958 
Type d-Just as an early onset paraplegia may rarely persist, after healing of the spinal lesion. in the presence of a persistent paravertebral abscess, so may a late onset paraplegia manifest itself for the first time under similar circumstances.

Significance of the type of paraplegia-Not only is the recognition of the type of paraplegia of more prognostic value than its grade but it may also have an important bearing on treatment. Thus, of five patients with paraplegia of type (a) three (grade 1, grade 1 and grade 2) recovered completely, one (grade 4) recovered incompletely with conservative treatment, and one (grade 1) recovered incompletely after costo-transversectomy and laminectomy.

One patient whose category was intermediate between types (a) and (b), with a grade 2 paraplegia of six weeks' duration that deteriorated to grade 4 , recovered completely after antero-lateral decompression.

There were two cases of type(b). One patient, with paraplegia that was originally of grade 3 , was treated conservatively and pursued a fluctuating course for two years before improving to the extent that her general condition was good, there were no flexor spasms, she had recovered full control of the sphincters and was learning to walk with calipers and crutches. The second patient, with paraplegia of grade 2, worsened temporarily after antero-lateral decompression, developing incontinence and shin sores, but within a month began to improve and made a partial recovery.

There were three cases of type (c). One patient with paraplegia of grade 2 worsened after antero-lateral decompression and later died; one whose condition had long fluctuated between grades 1 and 2 deteriorated permanently to grade 4 after antero-lateral decompression; and one with grade 3 paraplegia of fifteen months' duration temporarily regressed to grade 2 after antero-lateral decompression, but later relapsed and died with bedsores and toxaemia.

There were two cases of type (d). One patient with paraplegia of grade 3 recovered completely after antero-lateral decompression, and one with grade 1 paraplegia after evacuation of a large psoas abscess.

Of the thirteen patients with late onset paraplegia five were treated purely conservatively, and there were five antero-lateral decompressions, two costo-transversectomies and one laminectomy.

\section{OPERATIVE TREATMENT}

Costo-transversectomy-Of the twenty-seven costo-transversectomies, fifteen were carried out primarily on account of a large or tense paravertebral abscess, the radiological shadow of which persisted or was increasing despite adequate conservative measures, and not essentially for the relief of the paraplegia. Such an abscess, failing to regress with quiescence of the bone lesion, constitutes a potential source of recrudescence of disease or of its spread to adjacent vertebral bodies, and should be emptied irrespective of paralysis. In nine instances an abscess was encountered, but none was found in six. In three cases paraplegia was of grade 1 and in twelve of grade 2; all the patients later recovered completely with the exception of one with grade 2 paraplegia who exhibited slight residual paresis.

In twelve cases costo-transversectomy was performed for the relief of paraplegia. A grade 1 paraplegia of nearly three years' duration (abscess) and a paraplegia that had fluctuated between grades 1 and 2 for twenty months (no abscess) both recovered incompletely. Of four patients with grade 2 paraplegia of early onset one (abscess found) recovered completely but slowly, two (no abscess found) suffered permanent partial paraplegia and the fourth (abscess found) had a permanent complete paralysis. Of four patients with grade 3 paraplegias of early onset one (abscess found) was permanently paralysed, one (abscess found) was unimproved after costo-transversectomy but later recovered fully with antibiotics, one (abscess found) made a slow, full recovery, and one (abscess found) was unaffected and later died of cerebral malaria while improving after antero-lateral decompression. One grade 4 paraplegia (abscess) recovered incompletely, and one late onset paraplegia (no abscess) was permanent but partial. 
Although in some cases the result of costo-transversectomy may be dramatic, in only three cases did marked improvement follow so closely on costo-transversectomy as to be unequivocally attributable to it, and it must be considered an unreliable operation for the relief of paralysis.

The frequency with which no pus was found need cause no surprise, for a radiological shadow suggestive of an abscess may equally well represent an inflammatory mass or tuberculous granuloma containing little or no pus.

Laminectomy-This was performed in five cases, but in only one, that of a grade 4 paraplegia due to disease confined to the neural arches, was recovery so prompt and complete as to be unmistakably due to the laminectomy.

Antero-lateral decompression-Twenty-nine antero-lateral decompressions were carried out. Thirteen were in cases of grade 2 early onset paraplegia, and seven of these, with paralysis of four, two, two, two, three, five and six months' duration, and two with paralysis of unspecified but brief duration-nine in all-recovered completely. In five of these nine cases the indications for operation were: blistering of the feet with threatened sores; spinal luxation, anaesthesia with trophic sores, and flexor spasms (two cases). In four cases no clear indication is specified. In six of the nine cases marked improvement occurred within seven to ten days of operation and recovery was complete within two months. In one patient slight improvement, and in another moderate improvement, occurred within two weeks, and progressed to complete recovery within six months. In the last case there was marked improvement within a month, but the spinal lesion later relapsed and fluctuating minor signs persisted till healing of the lesion and final full recovery of the paraplegia occurred two years later. Of the four patients with grade 2 paraplegia that failed to recover completely one was unimproved and died suddenly five weeks later. A second was unaffected by antero-lateral decompression, later deteriorated, but recovered with residual weakness after later costo-transversectomy. The third failed to respond to decompression after twenty-three months of paraplegia and remained completely and permanently paralysed. The fourth case, one of long-standing grumbling disease with a grade 1 early onset paraplegia that had latterly deteriorated to grade 2 , was really comparable to a type (b) late onset paraplegia. There was no response to antero-lateral decompression and paralysis was permanent but incomplete.

Five operations were performed for grade 3 paraplegia of early onset. One patient with paraplegia of less than one month's duration recovered with slight residua, showing definite improvement within ten days of operation. Of three patients paralysed six weeks, two months and at least three months, the first improved within three weeks and recovered completely, the second with a progressively improving paraplegia died of cerebral malaria three months after operation and the third improved within two weeks and recovered with minor residual weakness. Lastly a paraplegia of fifteen months' duration, of grade 3 for at least five months, remained permanent and complete.

Of five grade 4 early onset paraplegias three (two of about a week's duration, one of two months' duration) recovered fully, improvement occurring in all within seven to fourteen days of operation. A child of one and a half years with grade 4 paraplegia associated with complete spinal luxation died from tuberculous meningitis with the paralysis unrelieved three months after operation; and a further grade 4 paraplegia of eight months' standing remained permanent and complete.

Six antero-lateral decompressions were performed for late onset paraplegia. The first case was that of a boy of seven with Pott's disease for three years, and a grade 2 paraplegia for a month finally deteriorating to grade 4 . At the onset of the disease there had been a paravertebral abscess, but when paraplegia supervened there was no gross kyphosis; radiographically there was evidence of activity, but no gross destruction and no paravertebral abscess. At operation the only evidence of activity was one small focus of carious, crumbly bone. Yet improvement occurred within a month and recovery was full. One type (b) case 
of two months' duration, which showed at operation much old caseous matter with some more recent, active, oedematous granulations, worsened temporarily after operation with incontinence and shin sores, but improved within a month to recover ultimately with marked residual weakness.

In the type (c) cases there was clinically, radiologically and at operation no sign of active disease, and in all there was very pronounced kyphosis. In one case of paraplegia of grade 2 an anterior bony ridge was removed, but paraplegia deepened and the patient died later with sores, emaciation and toxaemia. In a second case, with paraplegia that had long fluctuated between grades 1 and 2, the dural tube was at operation found flattened and stretched over the angulated anterior wall of the canal: no attempt was made to remove the bony ridge, but the paraplegia deepened permanently to grade 4 . In the third case, with grade 3 paraplegia of fifteen months' duration, improvement occurred after operation to the extent that the sores healed, flexor spasms were abolished, the patient was able to sit out in a chair and was measured for calipers, but later he relapsed and died with recurrent sores and toxaemia.

Lastly, a type (d) grade 3 paraplegia recovered completely. At operation a large paravertebral abscess was emptied, but no sign of active bone disease found, and it is probable that a costo-transversectomy would have served as well.

The first antero-lateral decompression in this series was performed in August 1952 and there were no operative or immediate post-operative deaths.

\section{DISCUSSION}

No mention has been made of damage to the cord by pathological dislocation. In six cases there was complete luxation, and in six severe subluxation, but, except in one case, this never seemed to be the dominant factor in the causation of an early onset paraplegia. In one case, in which the radiographic appearance suggested that the cord could scarcely have escaped transection, full recovery occurred after antero-lateral decompression.

It seems to be established, as Butler (1935) maintained, that the factors responsible for the development of an early onset paraplegia are the effects of adjacent inflammation, oedema and toxins on the cord, alone or abetted by mechanical compression from inflammatory granulations, debris or pus. As regards the latter, records of cerebro-spinal fluid examination were available in only three cases in this series. In two cases the fluid was clear and colourless, in one slightly turbid with formation of a web coagulum, and in all the pressure rose with jugular compression, the cell count was normal, the protein over 100 milligrams per 100 millilitres and the globulin increased suggesting partial block. Both the toxi-vascular and the mechanical compressive factors regress in most cases as the spinal bony lesion heals, and the paraplegia recovers accordingly. However, should this process be unduly delayed, recovery may be incomplete or the paraplegia remain permanent, and it is evident that if the acute recoverable changes in the cord are not relieved within a certain critical period, further changes ensue which are not reversible.

As Seddon (1935) stated, it is not the grade of paraplegia alone that determines the outcome but a combination of the grade and the duration. Taking the critical period for grade 2 paraplegias as six months, Seddon (1956) suggested that, once voluntary control has been lost, decompression should not be delayed longer than one month. He justified this apparent haste by pointing out that if the primary decompressive measure be a costo-transversectomy, four to eight weeks will elapse before its efficacy is known. If antero-lateral decompression should thereafter prove necessary, with a similar further recovery period, the critical period of six months will be nearing its close. The present series confirms that the critical period for almost certain full recovery is six months, although 80 per cent of the patients recovered completely after six to ten months' paraplegia. Moreover, of the forty-eight patients with grade 2 paraplegia that did recover fully, only twenty-four began to improve within three 
months of the onset. Thus, if Seddon's suggestion of operation within a month of onset is adopted, many patients would be subjected to unnecessary operation, and it must not be forgotten that most early onset paraplegias did recover with conservative measures alone even in pre-antibiotic days. Experience in this series suggests that in a case of early onset paraplegia of grade 2 it is expedient to pursue conservative measures for three months; if then there is no improvement and a large paravertebral abscess shadow is failing to decrease or is actually increasing, costo-transversectomy is performed. If pus is not encountered and released antero-lateral decompression is proceeded with at once. Except in the above circumstances conservative measures are persevered with for five months, and, if then there is no evidence of improvement, antero-lateral decompression is carried out. If at any time while under adequate conservative treatment a grade 2 paraplegia deteriorates or threatens to deteriorate to grade 3 or grade 4, antero-lateral decompression is carried out without delay.

Since it seems possible that there may be some advantage in establishing antibiotic control before operation, it is suggested that a patient admitted in a state of grade 3 or 4 paraplegia be given a preliminary trial of conservative treatment, including antibiotics, before operation. In such cases it is considered however that, in the absence of improvement, operative decompression should not be delayed longer than one month in grade 3 paraplegia, and two weeks in grade 4 paraplegia. Frequently the issue is not so clear-cut as suggested above, and careful judgment may have to be exercised in the case of a grade 3 or 4 paraplegia that has supervened on a grade 2 of variable duration, or a paraplegia that has fluctuated between grades 1 and 2 for perhaps two or three years.

Seddon (1935) stated that if there is still some voluntary control the spinal cord is in little danger of irrecoverable damage. Although this is generally true, permanent sequelae may occasionally result, and it is therefore suggested that operation be considered in any grade 1 paraplegia that has persisted for more than twelve months.

It seems, then, that, apart from some variation in opinion as to the time allowed to elapse before its employment, the indications for antero-lateral decompression in early onset paraplegia are fairly well defined and its results eminently satisfactory.

The prognosis in late onset paraplegia is notoriously less favourable, and the problems of treatment greater. At the one extreme the type (a) late onset paraplegia, with clear evidence of spinal activity and often a paravertebral abscess, may be treated on similar lines to the early onset case, the indications for operation being the same and the prognosis almost as favourable. In the type (b)case, however, activity is slow and grumbling, there is no paravertebral abscess, and even at operation one may encounter only minimal active granulation or oedema superimposed on areas of old crumbly, caseous or fibrous quiescent disease. This is in marked contrast to the florid reaction and paravertebral abscess of the early onset case, and it is not at once clear how such minimal activity could be capable of provoking a paraplegia at all. The answer seems to be that under the influence of years of slow, grumbling activity in its vicinity the substance of the cord has undergone some change, for long subclinical, and perhaps ischaemic or degenerative in nature. Thus, if a slight low-grade recrudescence of inflammation in the bony lesion should occur, it acts upon a damaged cord of low reserve.

In the type (c) case there is clinically, radiologically and at operation absolutely no evidence of active disease, so that local inflammation can be excluded as the precipitant cause of the paraplegia. At operation the anterior wall of the spinal canal is acutely angulated and the dura and its contained cord are often flattened and ribboned over this angulation, and it seems probable that stretching and friction of the cord over this bony spur are responsible for the paraplegia. Butler (1935) explained that this is not a primary stretching of an essentially normal cord over a bony spur, but that the cord has shrunk in length at the site of disease in consequence of the change in its substance postulated above-an irreversible change described by Seddon (1956) as a curious atrophy, an interstitial change the result of a chronic ischaemia, but not a gliosis-and so has become secondarily stretched over the angulation.

VOL. $40 \mathrm{~B}$, NO. 1, FEBRUARY 1958 
As regards treatment, the type (c) paraplegia caused by stretching of an irreversibly damaged cord over a bony angulation stands at the opposite extreme to the type (a) paraplegia which is the effect of adjacent inflammation on an essentially recoverable cord. Seddon (1956) emphasised the difficulties of removing such a solid, bony spur, and the dangers of inflicting further damage on the cord in the process. In one case in this series such damage resulted from the approach alone, without any attempt at removal of the bony ridge, possibly from interference with contributory vessels, either epidural or entering through the intervertebral foramina. Of the three patients with type (c) disease in this series one deteriorated and later died after removal of a bony ridge, a second deteriorated to a permanent grade 4 paraplegia after antero-lateral exposure without attempted attack on the spur, and the third, after a modest temporary improvement, relapsed and died some months later-a discouraging record. Although it is possible that more favourable results might be obtained with greater technical expertise, it seems probable that damage to the cord is in the main irreversible, and its residuum of functional tissue exceedingly vulnerable and precarious. Whereas little is to be lost by exploration if a type (c) paraplegia is complete already, I believe that in a case of partial paraplegia the chances of aggravation by operation outweigh those of benefit, and I prefer at present to eschew attempts to remove a bony ridge or to reroute the cord, and confine activities to a fairly extensive posterior fusion: it is hoped that the latter may obviate further increase in the gibbus and limit friction of the cord over the angulation, and so arrest the progress of the paraplegia.

The type (b) case falls intermediate between types (a) and (c), both cord degeneration and adjacent inflammation contributing their quota to the pathogenesis. Although there is some danger that disturbance of the damaged cord may intensify the paraplegia by further restricting its vascular supply, the benefit conferred by local decompression may well outweigh this risk. Some of these patients recover or improve considerably with conservative treatment, which, since the paralysis is often incomplete, may be given a long and generous trial. In these type (b) cases a gross kyphosis with extensive bony destruction often renders final healing protracted and uncertain, and it necessitates immobilisation of prohibitive duration. To facilitate healing and as a safeguard against relapse of the paraplegia, therefore, an extensive posterior fusion should always be undertaken once the paraplegia has responded either to conservative or operative measures.

Inter-body fusion at the time of antero-lateral decompression has not been attempted in this series because of the fear that inadequately fixed grafts might become dislodged and exert pressure on the cord.

In short, in cases of late onset paraplegia, the greater the activity of the bony lesion, the better is the prognosis, and the less are the risks of increased cord damage from antero-lateral decompression.

As regards the type of operation to be employed, laminectomy is desirable only in paraplegia from disease of the neural arches, or when disease of the cervical spine renders antero-lateral decompression inapplicable. Even when a large paravertebral abscess has been successfully evacuated, recovery from paraplegia after costo-transversectomy has proved most uncertain. When recovery does occur it is so late or protracted that it may seem to be coincidental, though undoubtedly a small proportion of prompt and dramatic results are obtained. The use of costo-transversectomy has, therefore, come to be restricted to three types of case: 1) cases of grade 2 early onset paraplegia that has persisted unimproved for three months, with a large paravertebral abscess that has failed to decrease or is increasing despite adequate conservative treatment; 2) the infrequent cases of paraplegia with a paravertebral abscess but without detectable (or with a minimal) bony lesion in the radiographs; 3) paraplegia persisting or arising for the first time with a long-standing residual paravertebral abscess which has persisted unchanged, although the spinal lesion is regressing, quiescent or even healed. 
In all other circumstances demanding decompression, the antero-lateral operation is chosen. Unless undue delay or long-standing disease has resulted in irreversible cord change, its results are reliable and usually evident within two weeks of the operation. At operation evacuation of any paravertebral abscess should be ensured. In one case in this series this was not done: improvement did not follow antero-lateral decompression, the paraplegia later deteriorated, and only recovered after costo-transversectomy.

\section{SUMMARY}

1. The results of treatment of 115 patients with Pott's paraplegia during a six and a half year period are reviewed.

2. The indications for operative intervention - in particular antero-lateral decompressionaccording to the duration, grade and type of paraplegia, are discussed.

3. No attempt has been made to justify the treatment of individual patients in the series, some of whom would undoubtedly have benefited from earlier operation; but lessons are pointed and conclusions drawn from the results, good and bad.

I wish to express my thanks to Sir Samuel Manuwa, Chief Medical Adviser to the Federal Government of Nigeria, for permission to publish this paper.

\section{REFERENCES}

Butler, R. W. (1935): Paraplegia in Pott's Disease, with Special Reference to the Pathology and Etiology. British Journal of Surgery, 22, 738.

Seddon, H. J. (1935): Pott's Paraplegia: Prognosis and Treatment. British Journal of Surgery, 22, 769.

Seddon, H. J. (1956): Pott's Paraplegia. In Modern Trends in Orthopaedics. (Second Series), p. 220. Edited by Sir Harry Platt. London: Butterworth \& Co. (Publishers) Ltd. 